\title{
Verzeichnis der vollständig oder im Auszuge mitgeteilten Aktenstücke.
}

\author{
Seite \\ Hertzberg an Podewils und Jacobi 17. 3. 1788 (Ausz.) $\ldots \ldots \ldots \ldots \ldots \ldots \ldots . \ldots 7$ \\ Friedrich Wilhelm II. an Jacobi 14. 10. $1788 \ldots \ldots \ldots \ldots \ldots \ldots \ldots \ldots \ldots \ldots$ \\ Jacobi an Friedrich Wilhelm II. 22. 10. 1788 (Ausz.) $\ldots \ldots \ldots \ldots \ldots \ldots \ldots$ 14-15 \\ Gutachten des Generals Möllendorff 17. Ir. 1788 (Ausz.) ............. 15-16 \\ Podewils und Jacobi an Friedrich Wilhelm II. 10. 1. 1789 (Ausz.)........ 88-89

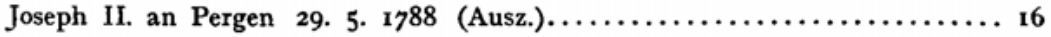

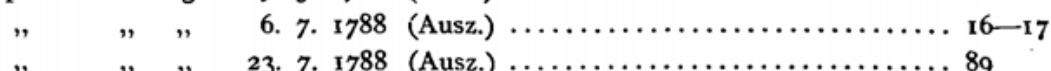

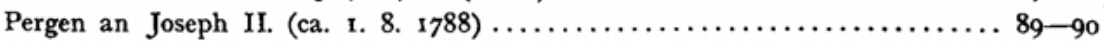

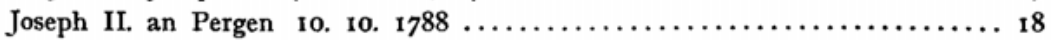

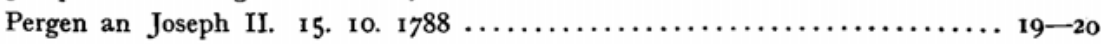

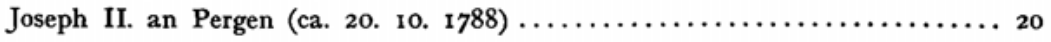

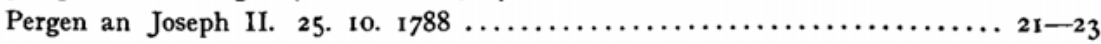

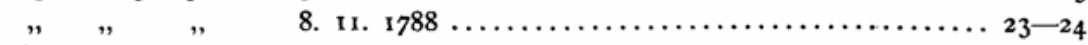

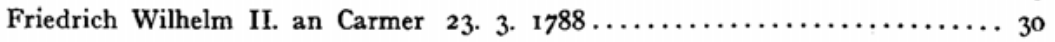

Fr. Rud. Grossing an Friedrich Wilhelm II. ca. 1. 5. $1788 \ldots \ldots \ldots \ldots \ldots \ldots, 30-32$

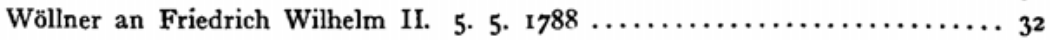

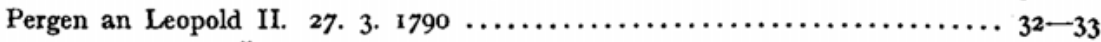

Fr. R. Grossing : Über Hungarns gegenwärtige Angelegenheiten (Ausz.) ..... 34-37

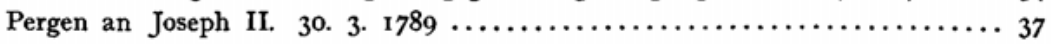

Jacobi an Friedrich Wilhelm II. 18. 2. 1789 (Ausz.) ...................... 92

Goertz an Karl August 5. und 26. 6. 1788 (Ausz.) .................. 92

Karl August an Karl Wilhelm Ferdinand von Braunschweig 26. 3. 1789.... 93

Karl Wilhelm Ferdinand an Hertzberg 4. 4. $1789 \ldots \ldots \ldots \ldots \ldots \ldots \ldots \ldots . \ldots \ldots$

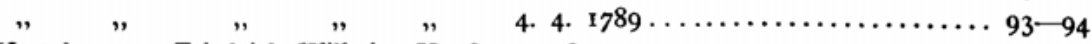

Hertzberg an Friedrich Wilhelm II. 8. 4. $1789 \ldots \ldots \ldots \ldots \ldots \ldots \ldots \ldots \ldots \ldots$

Friedrich Wilhelm II. an Hertzberg 10. 4. 1789 ................... 94-95

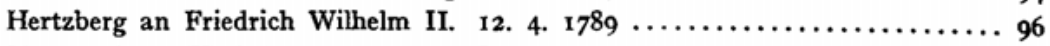

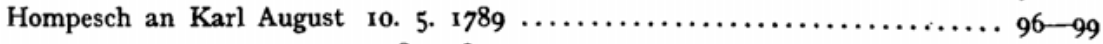

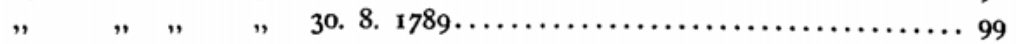

Hompesch sen. an Friedrich Wilhelm II. 22. 12. 1789.............. 99-100

Kabinettsministerium an Friedrich Wilhelm II. 6. 3. 1794 (Ausz.) ......... 100

Friedrich Wilhelm II. an Hertzberg 10. 4. $1789 \ldots \ldots \ldots \ldots \ldots \ldots \ldots \ldots$. $\ldots$.

Hertzberg an Friedrich Wilhelm II. 12. 4. 1789 ................... 100--104

Jacobi an Friedrich Wilhelm II. 7. 3. 1789 (Ausz.) .....................

$" \quad, \quad, \quad 6.6 .1789$ (Ausz.)...........................

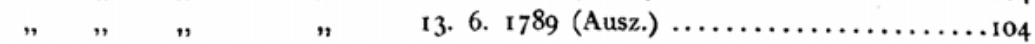


VI Verzeichnis der vollständig oder im Auszuge mitgeteilten Aktenstäcke.

Jacobi an Friedrich Wilhelm II. 17. 6. 1789 (Ausz.) .................. 105

$" \quad " \quad$ 31. 10. 1789 (Ausz.).................

Die Ungarischen Stände an Hertzberg (Mai 1789 ?).................. 47-48

$" \quad " \quad$ "Friedrich Wilhelm II. (Ausz.) $\ldots \ldots \ldots \ldots \ldots \ldots .48-49$

Jacobi an Friedrich Wilhelm II. 10. 10. 1789 (Ausz.) .................

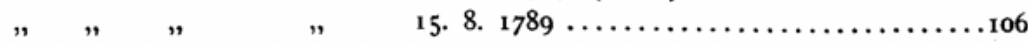

$" \quad " \quad$ "23. 10. 1789 (Ausz.) $\ldots \ldots \ldots \ldots \ldots \ldots \ldots \ldots$

Friedrich Wilhelm II. an Jacobi 28. 10. 1789 (Ausz.) $\ldots \ldots \ldots \ldots \ldots \ldots \ldots 106$

Jacobi an Friedrich Wilhelm II. 7. 11. 1789 (Ausz.) .................

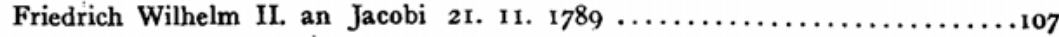

Weyland an Karl August 16. 3. 1790 (Ausz.) ......................

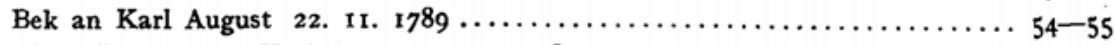

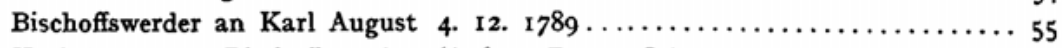

Karl August an Bischoffswerder (Anfang Dez. 1789) .................

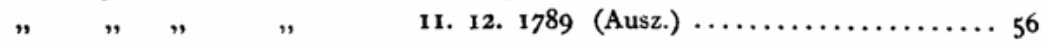

Bischoffswerder an Karl August 20. 12. 1789.................... 56-57

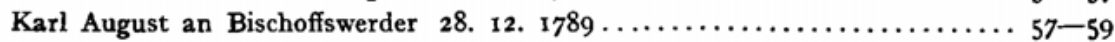

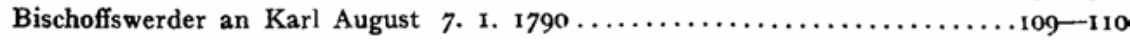

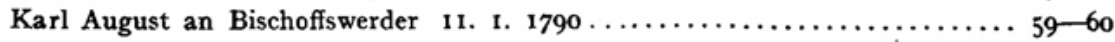

Bischoffswerder an Karl August 14. I. 1790...................... 61

Luise Auguste an Karl August 30. 1. 1790 (Ausz.) .................. 110

$" \quad, \quad, \quad "$ 4. 2. 1790 (Ausz.)...................110

Karl Wilhelm Ferdinand von Braunschweig an Karl August 10. 2. 1790 (Ausz.) 110

$" \quad " \quad, \quad, \quad, \quad " \quad$ (Febr. 1790) (Ausz.) I10-111

Stein an Karl August 8. 3. 1790 (Ausz.) ........................63 64

Jacobi an Friedrich Wilhelm II. 21. 10. 1789 (Ausz.) $\ldots \ldots \ldots \ldots \ldots \ldots \ldots$. $\ldots \ldots \ldots$

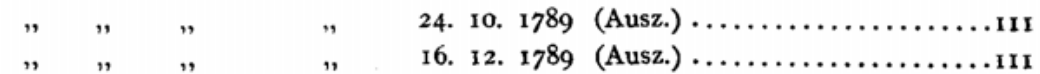

Podewils an Friedrich Wilhelm II. 19. I2. 1789 (Ausz.) $\ldots \ldots \ldots \ldots \ldots \ldots \ldots$ III

Jacobi an Friedrich Wilhelm II. 21. II. 1789 (Ausz.) $\ldots \ldots \ldots \ldots \ldots \ldots \ldots$ II2

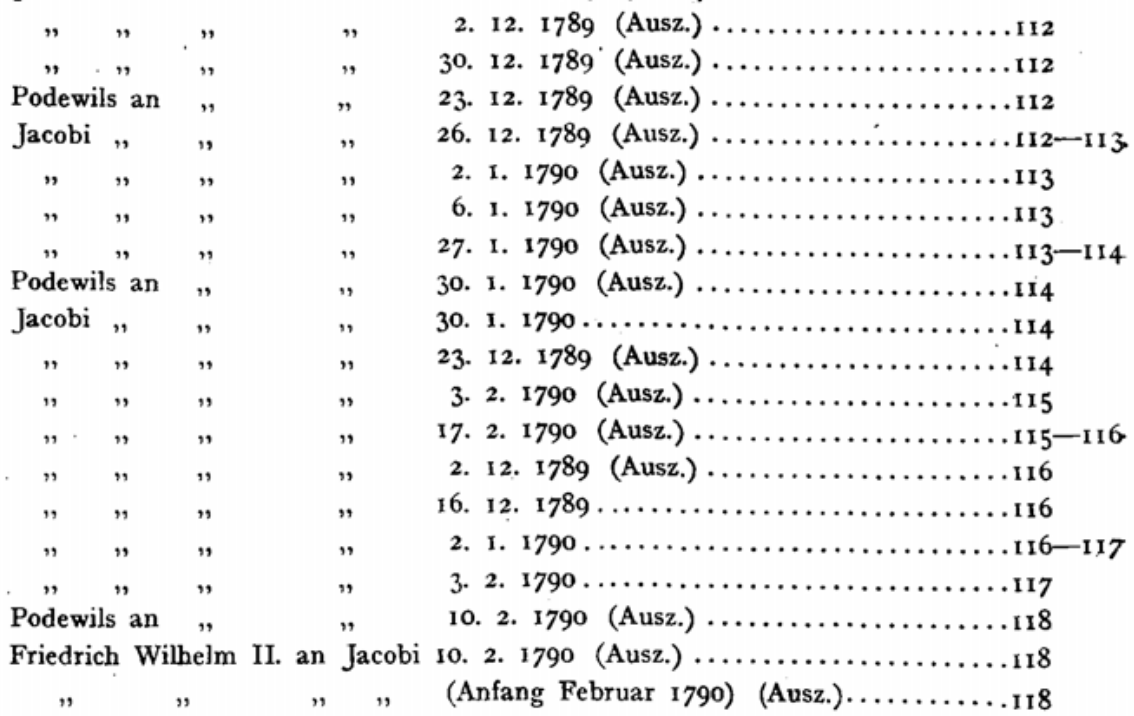




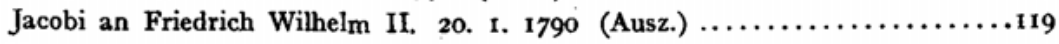

Karl Wilhelm Ferd. an Karl August 2. 2. 1790 (Aus\%.) . . . . . . . . . . . 119-120

Jacobi an Friedrich Wilhelm II. 20. 2. 1790 (Ausz.) ..............

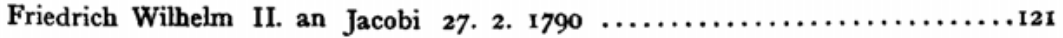

Jacobi an Friedrich Wilhelm II. 24. 2. 1790 (Ausz.) $\ldots \ldots \ldots \ldots \ldots \ldots \ldots \ldots \ldots$ I21-122

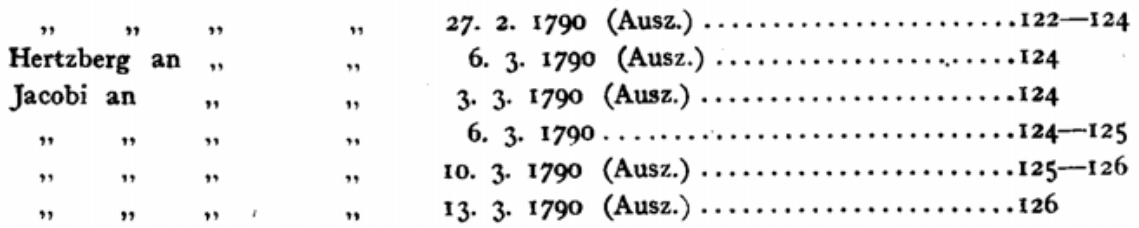

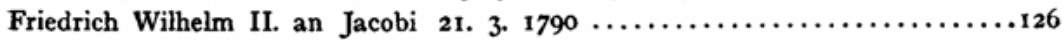

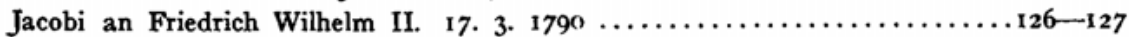

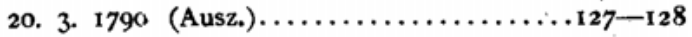

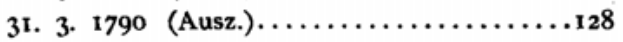

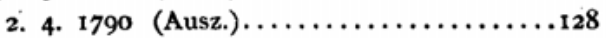

8. 5. 1790 (Ausz.)............... $128-129$

Schlabrendorff an Friedrich Wilhelm II. 10. 5. 1790 (Ausz.)........... 29

Jacobi an Friedrich Wilhelm II.

8. 5. 1790 (Ausz.)................ $129-130$

25. 5. 1790 (Ausz.) ................ 130

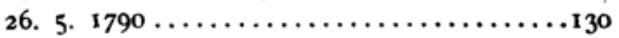

29. 5. 1790 (Ausz.) ................ 130-131

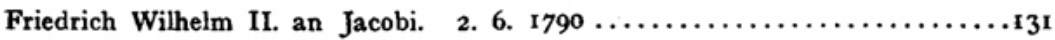

Jacobi an Friedrich Wilhelm II. 3. 6. 1790 (Ausz.) $\ldots \ldots \ldots \ldots \ldots \ldots \ldots \ldots$ I3

\begin{tabular}{|c|c|c|c|c|}
\hline " & $"$ & " & "' & 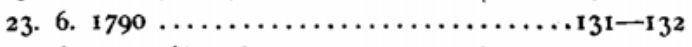 \\
\hline " & $"$ & $"$ & " & 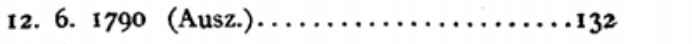 \\
\hline , & $"$ & " & $"$ & 26. 6. $1790 \ldots \ldots \cdots \cdots \cdots \cdots \cdots \cdots \cdots \cdots \cdots \cdots \cdots \cdots \cdots \cdots \cdots \cdots \cdots \cdots$ \\
\hline ", & ", & " & " & 6. 7.1790 (Ausz.) $\ldots \ldots \ldots \ldots \ldots \ldots \ldots \ldots \ldots \ldots \ldots \ldots \ldots$ \\
\hline
\end{tabular}

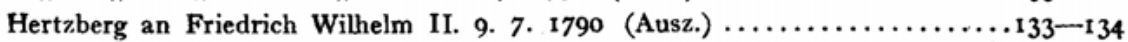
,
11. 7. 1790 (Ausz.)............ 134-135

, $\quad, \quad$ 27. 7. 1790 (Ausz.) $\ldots \ldots \ldots \ldots \ldots \ldots \ldots 135$

Friedrich Wilhelm II. an Hertzberg 12. 7. 1790 (Ausz.) ..............

Hertzberg an Friedrich Wilhelm II. 12. 7. 1790 (Ausz.).............

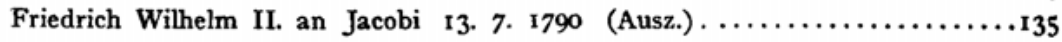

Jacobi an Friedrich Wilhelm II. 6. 7. 1790 (Ausz.) $\ldots \ldots \ldots \ldots \ldots \ldots \ldots \ldots$ 135-137

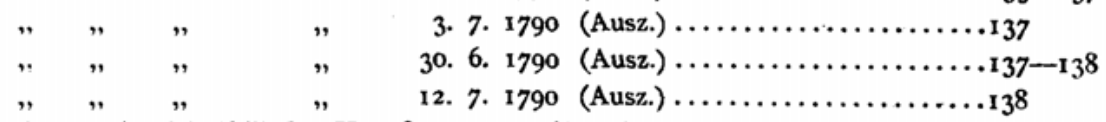

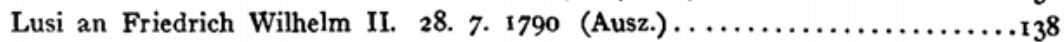

Jacobi an Friedrich Wilhelm II. 28. 7. 1790 (Ausz.) ...............

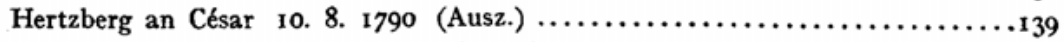

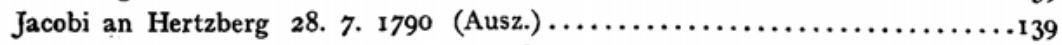

Podewils an Friedrich Wilhelm II. 21. 8.1790 (Ausz.) $\ldots \ldots \ldots \ldots \ldots \ldots \ldots \ldots$ 139-140

Jacobi an Friedrich Wilhelm II. 17. 7. 1790 (Ausz.) ...............

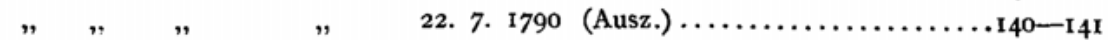

Podewils an Friedrich Wilhelm II. 18. 8. 1790 (Ausz.) .............

Jacobi an Friedrich Wilhelm II. 21. 8 . 1790 (Ausz.) $\ldots \ldots \ldots \ldots \ldots \ldots \ldots \ldots$ 142 
VIII Verzeichnis der vollständig oder im Auszuge mitgeteilten Aktenstücke.

Seite

Jacobi an Friedrich Wilhelm II. 27. 7. 1790 (Ausz.) $\ldots \ldots \ldots \ldots \ldots \ldots \ldots \ldots 142-143$

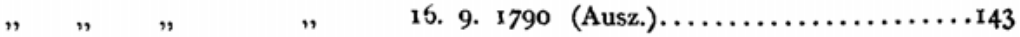

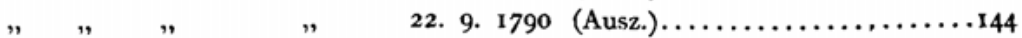

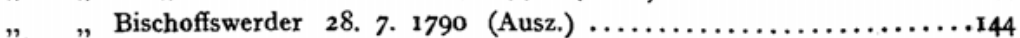

$" \quad$ Friedrich Wilhelm II. 21. 8. 1790 ......................

$" \quad$, Bischoffswerder 25. 8. 1790 (Ausz.) .....................

Hertzberg an Podewils 22. 10. 1790 (Ausz.) ....................... 45

Jacobi an Friedrich Wilhelm II. 30. 9. 1790 (Ausz.) ................. 145

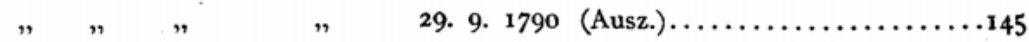

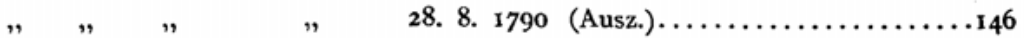

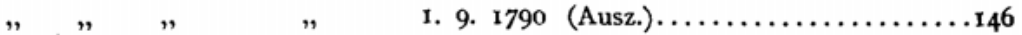

$" \quad " \quad, \quad 4.9 .1790$ (Ausz.).................146-147

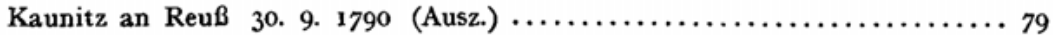

Jacobi an Priedrich Wilhelm II. 23. 9. 1790 (Ausz.) ................
", "
13. 10. 1790 (Ausz.)................147

Izdenczy an Joseph II. 8. 4. 1789 (Ausz.) .........................

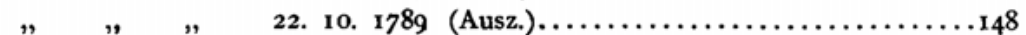

Joseph Grossing an Joseph II. 21. 10. 1789 (Ausz.) ................. 148-149

$" \quad, \quad$, Leopold II. 17. 2. 1791 (Ausz.) ................ 81-82

$" \quad " \quad, \quad$ (ungef. Juni 1791) (Ausz.) $\ldots \ldots \ldots \ldots \ldots 83$

$" \quad " \quad " \quad$ 31. 7. 1791 (Ausz.) $\ldots \ldots \ldots \ldots \ldots \ldots \ldots . \ldots 84$

Trenck an Forgách 6. 8. 1790 (Ausz.) ..........................

Joseph Grossing an Leopold II. 26. 8. 1791 (Ausz.) ................ 84-85

César an Friedrich Wilhelm II. 21. 9. I791 (Ausz.) ..................

Jacobi an Friedrich Wilhelm II. 8. 10. 1791 (Ausz.) ................ 149-1 50

Trenck an Friedrich Wilhelm II. 30. 1. 1792 ...................... 50

Jacobi an Friedrich Wilhelm II. 25. 10. 1790 (Ausz.)................

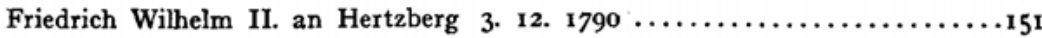

Jacobi an Friedrich Wilhelm II. 22. 10. 1791 (Ausz.) $\ldots \ldots \ldots \ldots \ldots \ldots \ldots \ldots 151$

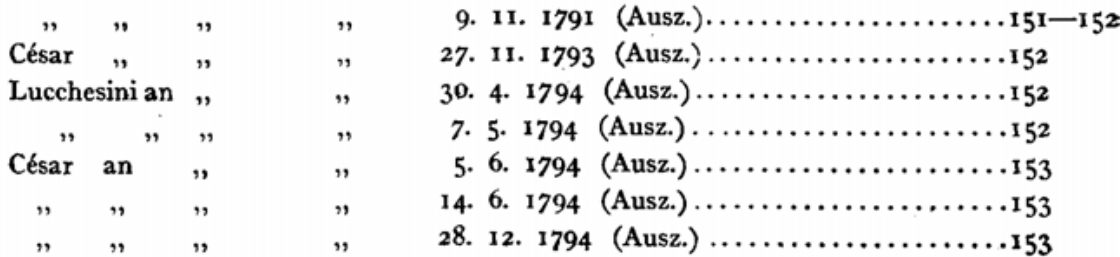

